\title{
Adoção de Tecnologia da Informação pelas Micro e Pequenas Empresas do Setor Hoteleiro de Sergipe
}

\section{Information Technology Adoption by Small and Medium Enterprises in the Hospitality Sector in Sergipe}

\section{Adopción de Tecnología de la Información por las Micro y Pequeñas Empresas del Sector Hotelero de Sergipe}

\author{
Wagner Amaral e Silva ${ }^{1}$ \\ Rivanda Meira Teixeira ${ }^{2}$
}

\begin{abstract}
Resumo: Grandes empresas do setor de turismo fazem o uso intensivo de Tecnologia da Informação (TI), também denominada Tecnologia da Informação e Comunicação (TIC), na promoção de seus produtos ou serviços e essa tendência também tem sido incorporada pelas micro e pequenas empresas (MPE) do setor hoteleiro. Para melhor compreender tal fenômeno, este estudo tem o objetivo de analisar os fatores que afetam a adoção de TI pelas MPEs do setor hoteleiro do estado de Sergipe. Possui abordagem qualitativa e utiliza como estratégia de pesquisa a de estudo de casos múltiplos. Verificou-se que as empresas hoteleiras que participaram do estudo possuem computadores e todos são conectados à Internet. Verificou-se também que a indisponibilidade da TI tem impacto negativo nos negócios. Identificou-se diferentes perfis de gestores e observou-se que o conhecimento em informática dos funcionários é indispensável. As limitações deste estudo são inerentes à estratégia de estudos de caso.
\end{abstract}

Palavras-Chave: Tecnologia da Informação. Adoção. Turismo. Setor Hoteleiro. Micro e Pequenas Empresas.

Abstract: Tourism big companies make extensive use of Information Technology (IT), also named Information and Communication Technology (ICT), to promote their products or services and this trend has also been incorporated by Small and Medium Enterprises (SME) from the hospitality sector. This study intend to increase the understanding of this phenomenon in order to analyze IT adoption by SME businesses of hospitality industry examined exclusively in the state of Sergipe. It was used a qualitative approach with a multiple case study strategy. It has been found that all hospitality enterprises analyzed own computers and they are all connected to Internet. It has also been found that IT failure has a high impact. Different managers' profiles had been identified and it was observed that staff's IT knowledge is considered indispensable. Limitations of this research are directly connected to the limits of multiple case study strategy.

1 Bacharel em Ciência da Computação e Mestrando em Administração de Empresas pela Universidade Federal de Sergipe E-mail: silvaw.br@gmail.com

2 Mestrado em Administração pela COPPEAD/UFRJ (1979). Doutorado em Administração pela Cranfield University Inglaterra (1996). Pós Doutorado em Gestão Turismo na Bournemouth University, Inglaterra e na Strathclyde University Escócia (20002001). Pós Doutorado em Empreendedorismo em Turismo na HEC em Montreal, Canadá (2007). Coordenadora do Mestrado em Administração da UFS, Professora dos Mestrados em Administração e Economia da UFS. E-mail: rivandateixeira@gmail.com 
Keywords: Information Technology. Adoption. Tourism. Hospitality Industry. Small and Medium Enterprises.

Resumen: Las grandes empresas de turismo presentes en el mercado hacen el uso intensivo de Tecnología de la Información (TI), también denominada Tecnología de la Información y Comunicación (TIC), en la promoción de sus productos o servicios, y esa tendencia también tiene sido incorporada por las micro y pequeñas empresas (MyPEs) del sector hotelero. Para mejor comprender tal fenómeno, se propone este estudio con el objetivo de analizar los factores que afectan la adopción de TI por las MyPEs del sector hotelero del estado de Sergipe. Posee abordaje cualitativa y utiliza como estrategia de pesquisa casos prácticos. Se verificó que los medios de hospedaje participantes poseen ordenadores, y todos son conectados a la Internet. Se verificó también que el impacto de la indisponibilidad de TI fue considerado alto. Se identificó diferentes perfiles de gestores y se observó que el conocimiento en informática de los funcionarios es indispensable. Las limitaciones de este estudio están ligadas directamente a las limitaciones de la estrategia de casos prácticos.

Palabras-claves: Tecnología de la Información. Adopción. Turismo. Industria Hotelera. Micro y Pequeñas Empresas.

\section{INTRODUÇÃO}

A Tecnologia da Informação (TI), também denominada Tecnologia da Informação e Comunicação (TIC), vem se tornando, cada vez mais, parte integrante das ferramentas de gestão das organizações em razão da dependência cada vez maior de informação e conhecimento. As grandes empresas fazem o uso intensivo de TI na promoção de seus produtos ou serviços, e essa tendência também tem sido incorporada pelas micro e pequenas empresas (MPE).

As MPEs são identificadas como um dos principais provedores da economia dos países, destacandose principalmente pelo fomento do Produto Interno Bruto (PIB) e pela geração de empregos (SEBRAE, 2012). Ainda segundo o SEBRAE (2012), Serviço Brasileiro de Apoio a Micro e Pequena Empresa, o salto econômico vivenciado pelo Brasil na última década tem facilitado que essas empresas sobrevivam e se tornem mais competitivas.

As diversas mudanças no mercado impulsionaram a adoção de $\mathrm{Tl}$ e impactaram diretamente as MPEs, principalmente nos setores que fazem uso intensivo de informações, que é o caso da indústria turística (Buhalis \& Law, 2008; Farias et al., 2011). A gestão dessas organizações está se tornando cada vez mais complexa, e a TI tornou-se uma das principais ferramentas para as áreas de planejamento, gestão e marketing, inclusive no estreitamento das relações com clientes e no desenvolvimento de novos negócios e parcerias (Buhalis \& Law, 2008; Zagheni \& Luna, 2011; Nandan, 2009).

O turismo receptivo no Brasil não é tão expressivo se comparado com países desenvolvidos. Por exemplo, enquanto no Brasil tivemos 5,2 milhões de turistas estrangeiros em 2010, a França foi visitada por 76,8 milhões, e países como Estados Unidos, China e Espanha receberam entre 50 e 60 milhões (IBGE, 2012). No entanto, nessa década, o Brasil deve ter essa situação alterada em função dos eventos esportivos da Copa do Mundo de Futebol em 2014 e Jogos Olímpicos em 2016.

Um dos pilares do setor turístico são os meios de hospedagem, pois são os responsáveis por acomodar os turistas e, dessa forma, tem forte influência na avaliação final da experiência vivida na viagem. Por isso, é importante que os hotéis e pousadas se adequem aos padrões dos potenciais turistas que irão visitar o Brasil durante os próximos anos. É mister destacar também que não só as cidades sede 
serão favorecidas pela demanda turística. As empresas do setor hoteleiro em Sergipe, particularmente, poderão se beneficiar desses eventos em razão de suas belas praias e de suas riquezas culturais.

Apesar de ainda não ser um dos principais destinos turísticos, Sergipe já vem se destacando no cenário nacional com relação ao aumento da demanda turística. Em janeiro de 2012, a média registrada pelos meios de hospedagem atingiu 92\% de ocupação (ABIH/SE, 2012). O estado de Sergipe ainda possui um potencial de crescimento turístico grande porque seu fluxo turístico é, majoritariamente, intrarregional $(60,4 \%)$. Os estados da Bahia e de São Paulo são responsáveis por 49,2\% dos turistas que visitaram o estado, destes 31,5\% são baianos e 17,7\% paulistas (EMSETUR, 2012).

A despeito da crescente importância do setor turístico e consequente aumento do uso de TI pelas micro e pequenas empresas, a maioria das pesquisas relacionadas à adoção de $\mathrm{TI}$ são realizadas em empresas de grande porte (Nandan, 2009; Yang \& Fu, 2008). Além disso, a TI auxilia na promoção dos destinos turísticos, e as MPEs precisam estar atentas ao número crescente de turistas que planejam suas viagens através da Internet (Marujo, 2008; Thomaz et al., 2013). Ressaltasse-se ainda que os meios de hospedagem não aproveitam todo o potencial disponibilizado pela TI (Crispim \& Dultra, 2005; Abreu \& Baldanza, 2009).

Este estudo tem como o objetivo analisar os fatores que afetam a adoção de TI pelas micro e pequenas empresas do setor hoteleiro do estado de Sergipe. Especificamente pretende-se: (1) verificar os equipamentos e ferramentas de TI existentes nas MPEs do setor hoteleiro de Sergipe; (2) analisar a dependência do negócio com relação à Tl; (3) analisar a experiência dos gestores dessas empresas com relação à TI e; (4) identificar as principais dificuldades enfrentadas na adoção de TI pelas MPEs do setor hoteleiro de Sergipe. Para isso, utiliza-se a abordagem qualitativa e a estratégia de pesquisa de estudo de caso múltiplos, a partir de evidências coletadas em entrevistas semiestruturadas e fontes documentais.

Importa destacar as contribuições acadêmicas deste estudo para o campo do turismo. O setor de turismo encontra-se em expansão e consolidação, possuindo peculiaridades que o diferenciam de outras atividades econômicas tradicionais. Essa pesquisa se justifica como ferramenta de construção de conhecimento, considerando que poucos estudos analisaram a adoção de TI pelas micro e pequenas empresas, tendo a atividade turística como objeto. Além disso, é notório o destaque crescente da adoção e uso de ferramentas de TI pelos empreendimentos turísticos, em especial pelos meios de hospedagem.

Além desta introdução, o estudo está organizado em cinco partes. Inicialmente, será apresentada uma revisão bibliográfica da (i) adoção de TI nas MPEs e da (ii) TI no setor de turismo. Em seguida, detalhase a metodologia e a seguir os casos são analisados comparativamente. Enfim, o estudo é concluído com a apresentação de suas implicações e limitações.

\section{ADOÇÃO DE TECNOLOGIA DA INFORMAÇÃO NAS MICRO E PEQUENAS EMPRESAS}

Pesquisas sobre adoção de TI podem ser realizadas e classificadas em dois níveis, individual ou organizacional (Yang \& Fu, 2008). Segundo estes autores, no nível individual, as pesquisas tem como objetivo verificar o uso e a aceitação de tecnologias por indivíduos nas empresas. No que concerne ao organizacional, existem pesquisas que analisam variáveis estratégicas, financeiras e ambientais.

A maioria das pesquisas relacionadas para a adoção de TI é realizada em empresas de grande porte (Nandan, 2009; Yang \& Fu, 2008). Segundo Yang e Fu (2008), a adoção de tecnologias nas micro e pequenas empresas diferem das grandes corporações em razão de suas particularidades como, por exemplo, o tamanho da empresa e suas características econômicas. 
Conforme o SEBRAE (2012), as micro e pequenas empresas representam 99\% do total de empresas em atividade no Brasil, empregam formalmente $51,6 \%$ dos brasileiros e têm participação em torno de $40 \%$ da massa salarial nacional. Além da sua relativa representatividade, as micro e pequenas empresas são responsáveis por $12,7 \%$ do PIB nacional (SEBRAE, 2012). Em função da sua relevância, é preciso que tais empresas se fortaleçam e se mantenham competitivas. Para isso, é importante que as MPEs ofereçam serviços ou produtos especializados para seus clientes, o que pode ser auxiliado pela adoção de tecnologia da informação.

Nesse sentido, a adoção de TI em uma organização pode influenciar no seu desempenho e no crescimento por meio da melhoria da produtividade, competitividade, eficiência e eficácia (Hameed \& Counsell, 2012). Pode também promover o acesso a novos mercados (Nandan, 2009), além de facilitar a gestão sustentável, a integração de processos e a coordenação da produção (GHOBAKHLOO et al., 2012). Para atingir tais objetivos, indica-se que os investimentos para a adoção de tecnologias sejam planejados, pois os ganhos percebidos por empresas que planejam a adoção são superiores aos daquelas que não o fazem (Lunardi et al., 2010). Decisões sem prévia avaliação podem levar a estratégias de investimento deficientes e imprecisas em TI, o que pode pôr em risco a sobrevivência do negócio (Ghobakhloo et al., 2012).

Em virtude da popularização dos microcomputadores a partir da década de 1980, tecnologias como hardware e software tiveram seus custos reduzidos, o que facilitou o acesso, a adoção e o uso de tecnologia da informação por MPEs. Porém, muitas vezes, a falta de conhecimento em Tl ou a preocupação com questões financeiras pode impactar na não adoção ou numa adoção lenta. Muitas empresas desse porte aguardam que outras adotem a tecnologia antes para que possam observar a viabilidade e os resultados financeiros com o objetivo principal de verificar se o retorno sobre o investimento ultrapassa os custos de adoção das novas tecnologias (Dholakia \& Kshetri, 2004).

A Internet tornou-se acessível uma década após os microcomputadores, o que facilitou o acesso a informações por clientes e organizações, e a comunicação entre estes. Zilber e Araújo (2012) realizaram um estudo em pequenas empresas num país emergente da América Latina que adotaram TI na realização de suas atividades e concluíram que o principal valor entregue ao adotar e utilizar TI, em especial a Internet, é a melhoria no reconhecimento da marca e do produto/serviço das empresas. Mesmo assim, por causa principalmente da limitação de recursos, existe receio em sua adoção por parte das MPEs. Nessas empresas, segundo Dholakia e Kshetri (2004), a adoção de Tl é gradual, pois existem diversos estágios até que exista uma decisão favorável à sua adoção.

As oportunidades e ameaças no ambiente competitivo, os pontos fortes e fracos decorrentes de aspectos organizacionais, as características específicas da tecnologia e as características do proprietáriogerente destacam-se como principais fatores que influenciam na adoção de novas tecnologias em micro e pequenas empresas, porém a velocidade com que essas tecnologias são adotadas é comprometida pela escassez de recursos humanos capacitados e de recursos financeiros (Gargallo-Castel \& Ramírez-Alesón, 2007). Em diversos estudos, as características do proprietário são enfatizadas em razão do seu maior poder de decisão em micro e pequenas empresas (Gargallo-Castel \& Ramírez-Alesón, 2007; Grandon \& Pearson, 2004; Hameed \& Counsell, 2012; Zilber \& Araújo, 2012; Ghobakhloo et al., 2012).

O fator determinante para que o proprietário ou gerente seja a favor ou não da adoção de TI pelas empresas é a sua percepção das características, oportunidades e ameaças presentes em cada tecnologia (Gargallo-Castel \& Ramírez-Alesón, 2007). Os gestores devem ser capazes de avaliar a relação custobenefício da adoção de TI (Ghobakhloo et al., 2012), ou seja, é preciso que o gestor tenha a percepção do 
valor estratégico resultante da implementação e do uso de tecnologia (Grandon \& Pearson, 2004). Muitas vezes essa percepção é dificultada porque os benefícios que a TI pode proporcionar não são totalmente claros para os micro e pequenos empresários (Lunardi et al., 2010), o que pode resultar numa fraca influência da decisão dos proprietários-gerentes para a adoção de TI (Hameed \& Counsell, 2012).

Ma, Buhalis e Song (2003) identificaram quatro estágios geralmente presentes na adoção de TI pelas organizações: processamento de dados, gestão, estratégia e rede. No primeiro estágio, a organização utiliza a TI apenas para comunicação e acesso a informações. No estágio de gestão, a Tl é empregada no controle das principais atividades da organização. Já no estágio de estratégia, as ferramentas tecnológicas fornecem apoio estratégico. Enquanto, no estágio de rede, a TI integra toda a cadeia de valor da empresa.

\section{TECNOLOGIA DA INFORMAÇÃO NO SETOR DE TURISMO}

O setor de serviços, por ter características heterogêneas, é importante tanto para a economia nacional como para a mundial, sendo reconhecido pela sua elevada geração de empregos, o que contribui em grande medida para a composição do PIB dos países devido à geração de valor e renda na economia (IBGE, 2010). O turismo se caracteriza como importante atividade sociocultural e é por meio dele que um país ou uma região realiza a promoção de suas particularidades, principalmente de sua cultura e de sua natureza (IBGE, 2012).

Para o crescimento do turismo brasileiro, terá de haver um maior alinhamento entre o poder público e a iniciativa privada para suportar a demanda e, com isso, incentivar a captação de novos turistas. Neste contexto, a TI pode ser inserida como uma aliada dos governos e organizações em virtude do suporte que pode prestar para atividades como gestão, planejamento e marketing. A TI tornou-se uma parceria ideal para o turismo por não oferecer ameaças às áreas humanas das quais o turismo é dependente, seja ela sociocultural, econômica ou política (Poon, 1988), e por permitir que as organizações desta indústria comuniquem-se instantaneamente com clientes e fornecedores em todo o mundo (Buhalis, 2003 apud Farias et al., 2011).

Por outro lado, apesar de reconhecem que o aparato tecnológico que vem sendo utilizado pelo turismo promove o desenvolvimento econômico, Cacho e Azevedo (2010) alertam sobre questões socioambientais e culturais, já que algumas áreas na cadeia produtiva do turismo são apropriadas ou totalmente modificadas após a implantação de novas tecnologias. Bezerra, Luft e Dacorso (2012) destacam que os impactos negativos decorrentes da adoção de novas tecnologias no turismo podem ser reduzidos a partir da adaptação do modelo de dinâmica da inovação para guiar políticas de gestão e de planejamento.

O novo ambiente de negócios influenciado pela TI demandou mudanças em inúmeros setores, inclusive nas empresas do setor turístico (Buhalis \& Law, 2008; Farias et al., 2011). A implantação de serviços on-line possibilitou maior contato com o cliente, contudo provocou o surgimento de diversas concorrências anteriormente inexistentes entre parceiros, como é o caso dos fornecedores de transporte, das agências de viagens e das operadoras de turismo (Poon, 1988).

Paradoxalmente, a cooperação entre diferentes fornecedores também foi incentivada por meio dos portais que proporcionam aos consumidores a capacidade de pesquisar e adquirir on-line todos os serviços de uma viagem a partir de um único site (O'Connor \& Frew, 2002). Assim, os turistas ganharam maior facilidade no acesso à informação e adquiriram maior poder de independência na hora de escolher o destino turístico desejado (Cacho \& Azevedo, 2010; Flores et al., 2012; Thomaz et al., 2013). Atualmente, o turista é capaz de planejar toda a sua viagem, desde passagens e local de hospedagem até os dias e 
horários de visitas a locais turísticos. Com isso, aumentam as possibilidades de oferta de diversos serviços turísticos por meio da $\mathrm{TI}$, com destaque para a Internet.

O ambiente virtual surge como mais uma opção de aquisição de produtos e serviços turísticos pelos consumidores (Abreu \& Baldanza, 2009). Os serviços turísticos on-line são intangíveis e necessitam criar percepções emocionais, principalmente quando a viagem apresenta caráter hedônico (Farias et al., 2011). Além disso, os clientes procuram por comodidade e rapidez (Raposo \& Pereira, 2012). Assim, é essencial prover serviços eficientes para motivar os clientes em suas escolhas ( $O^{\prime}$ Connor \& Frew, 2002). As informações disponíveis para os turistas tendem a melhorar a qualidade do serviço, desde que sejam utilizadas de maneira oportuna e precisa para que se tornem relevantes e levem à satisfação do cliente (Buhalis, 1998).

Porém, apesar do reconhecimento da Internet como um meio de comunicação fundamental para a atividade turística, diversas organizações ainda apresentam deficiências em seus portais e, consequentemente, na promoção de sua localidade ou de sua região como destino turístico (Marujo, 2008). Os gestores precisam estar atentos ao volume de informações apresentadas às pessoas que irão adquirir seus produtos e serviços. As informações sobre o destino turístico devem ser suficientes para que o turista tenha a segurança necessária no momento da compra, principalmente por causa do crescimento de viajantes independentes e do surgimento de novas mídias de compartilhamento de informações na Internet (Thomaz et al., 2013).

Dessa forma, o crescimento da oferta e da heterogeneidade de serviços podem provocar o surgimento de insegurança nas operações realizadas no ambiente virtual, principalmente no que toca aos riscos financeiros. Alguns turistas têm procurado reduzir esses riscos por meio da experiência própria de uso ou de pares e de garantias financeiras (Souza et al., 2012).

Todavia, os usuários têm se mostrado cada vez mais insatisfeitos com os serviços off-line e os têm preterido pelos serviços on-line (Gouvêa et al., 2012). Nesse sentido, Barbosa, O'Neil e Marins (2005) identificaram que numa amostra de 113 turistas ingleses no Brasil, 68,8\% deles utilizaram a Internet para realizar o planejamento de sua viagem e apenas $18,8 \%$ usaram os serviços de um agente de viagens.

Um dos grandes beneficiados pelas mudanças ocorridas no setor turístico são os meios de hospedagem. Porém, estas empresas não aproveitam todo o potencial disponibilizado pela TI (Crispim \& Dultra, 2005; Abreu \& Baldanza, 2009). Os sistemas e equipamentos precisam estar configurados de forma que impulsionem benefícios na gestão e na promoção do empreendimento, o que demanda uma atenção especial dos gestores.

Os softwares de gestão hoteleira apresentam vantagens por proporcionar um maior armazenamento e controle de informações, principalmente ligadas às áreas financeira e de logística. Já as ferramentas relativas à promoção estão integradas diretamente com a Internet. No modelo atual de gestão dos empreendimentos hoteleiros, a conexão dos meios tecnológicos e informacionais resultam em técnicas mais eficientes e baratas para a criação de elos com os potenciais clientes (Chagas et al., 2013).

Contudo, as poucas informações fornecidas pelos hotéis e pousadas são exploradas apenas nos sites dessas empresas como mero mecanismo de difusão de informações e promoção turística (Crispim \& Dultra, 2005), o que caracteriza uma significativa deficiência em relação aos recursos técnicos e de comunicação possibilitados pela web (Farias et al., 2011). Mondo, Santos e Costa (2010) e Raposo e Pereira (2012) destacam a necessidade de conhecimento dos gestores acerca do comportamento e da percepção de importância dos consumidores com relação às ferramentas de marketing utilizadas pelos hotéis para que possam evoluir em busca da melhoria contínua dos serviços on-line. Contudo, Barbosa, Souza, Farias e 
Kovacs (2013) denotam que o que se percebe é um excesso de padronização de conteúdo, dificultando a diferenciação dos atrativos e particularidades de cada estabelecimento.

O marketing digital tem desempenhado um papel importante na promoção da linha de turismo. Assim, meios de divulgação como redes sociais e sites de busca devem ser mais explorados pelas organizações do setor hoteleiro (Thomaz et al., 2013). As redes sociais proporcionam a captação de potenciais clientes de maneira efetiva e barata sem a necessidade de aquisição de novas ferramentas ou equipamentos (Kasavana et al., 2010 apud Atadil et al., 2010). Atadil et al. (2010) verificaram que 70\% das pessoas estão procurando informações sobre hotéis dos seus destinos turísticos nas redes sociais, pois estas destacam-se por sua interatividade e pelo compartilhamento, confiança e utilidade das informações.

Os hotéis e pousadas necessitam continuar a investir na adoção de $\mathrm{TI}$, principalmente no que tange ao serviço de reserva e às atividades de gestão e estratégia. Além do próprio site, essas empresas precisam ampliar a cobertura dos serviços por meio de outros mecanismos na web para adquirir o máximo de vantagens proporcionadas por essas ferramentas (O'Connor \& Frew, 2002).

\section{METODOLOGIA}

Este estudo se situa no paradigma interpretativista ou fenomenológico, que permite que o pesquisador analise e tente entender as diferentes construções e significados a partir dos comportamentos e das experiências vividas pelos atores humanos (Easterby-Smith et al., 1999; Saunders et al., 2007). Utilizase uma proposta exploratória porque se deseja esclarecer e ampliar a compreensão de um determinado problema, e descritiva em razão da representação de detalhes específicos de uma situação (Saunders et al., 2007; Neuman, 1997).

Utiliza-se como estratégia de pesquisa o estudo de caso múltiplo, que é empregado quando se deseja dispor de um conjunto de casos para o melhor entendimento de um fenômeno e para a melhor teorização possível (Stake, 1994). Estudos de caso múltiplos baseiam-se na lógica de replicação (generalizações analíticas), que tem o objetivo de generalizar um conjunto singular de resultados para uma teoria mais abrangente (Yin, 2001).

O estudo foi realizado com empresas do setor hoteleiro do estado de Sergipe classificadas como micro ou pequena empresa segundo critério do Sebrae (SEBRAE, 2012), o qual indica que microempresas possuem até nove funcionários e pequenas empresas têm de dez a 49 empregados. As empresas selecionadas se destacam por sua localização e relevância para o turismo do estado.

Com relação à validade externa (Yin, 2001), foi elaborado e seguido um protocolo para proceder a esta pesquisa. Já no que tange à validade do constructo, este estudo utilizou fontes múltiplas de evidências, quais sejam entrevistas e fontes documentais. Além disso, a transcrição das entrevistas foi revisada pelos participantes da pesquisa.

Para a coleta de dados foi elaborado roteiro da entrevista com base nas categorias analíticas e elementos de análise que estão apresentados no Quadro 1.

Quadro 1 - Categorias analíticas e elementos de análise

\begin{tabular}{|l|l|}
\hline Categorias & Elementos de Análise \\
\hline Equipamentos e ferramentas de TI existentes & - Equipamentos de TI implantados \\
(Zilber \& Araújo, 2012) & - Ferramentas de TI instaladas \\
\hline
\end{tabular}




\begin{tabular}{|l|l|}
\hline \multirow{2}{*}{ Dependência da TI (Ma et al., 2003) } & $\begin{array}{l}\text { - Acesso a informações chave para o negócio } \\
\text { - Disponibilização de informações para clientes } \\
\text { - Possibilidade de realizar novos negócios } \\
\text { - Novos canais de comunicação com clientes }\end{array}$ \\
\hline $\begin{array}{l}\text { Experiência dos gestores com TI (Grandon \& } \\
\text { Pearson, 2004) }\end{array}$ & $\begin{array}{l}\text { - Conhecimento do gestor com relação à TI } \\
\text { - Envolvimento do gestor nos processos de adoção }\end{array}$ \\
\hline $\begin{array}{l}\text { Dificuldades na adoção de TI (Gargallo-Castel \& } \\
\text { Ramírez-Alesón, 2007) }\end{array}$ & $\begin{array}{l}\text { - Escassez de Recursos Financeiros } \\
\text { - Falta de mão-de-obra qualificada } \\
\text { - Resistência a mudanças }\end{array}$ \\
\hline
\end{tabular}

Foram utilizadas entrevistas semiestruturadas, que se caracterizam por possuir uma lista de perguntas abertas. No entanto, essas perguntas podem não obedecer uma ordem pré-estabelecida, assim como se pode omitir ou adicionar perguntas para melhor explorar os objetivos do estudo de acordo com as particularidades de cada organização (Saunders et al., 2007).

A análise dos dados foi realizada com a técnica de análise de conteúdo, que consiste num conjunto de técnicas que objetivam obter a estrutura e os elementos de fontes de dados para esclarecer suas diferentes características (Laville \& Dionne, 1999; Bardin, 1977 apud Vergara, 2008).

\subsection{Análise Comparativa dos Casos}

Após a descrição individual de cada caso com base nas categorias analíticas, foi realizada a análise comparativa dos casos estudados. Eisenhardt (1989) destaca que um passo fundamental nesse processo é o conhecimento da realidade de cada um dos casos em particular, ou seja, é preciso que o pesquisador se torne "intimamente familiarizado com cada caso como uma entidade autônoma" (Eisenhardt, 1989, p. 540). Além disso, a autora afirma que é preciso examinar os dados de diversas maneiras divergentes. Assim, é possível realizar comparações mais rápidas e eficazes ao analisar os casos em conjunto (cross-case analysis).

\subsubsection{Características das empresas e dos entrevistados}

Na Tabela 1, são apresentadas as características das empresas hoteleiras analisadas com relação ao local, número de funcionários e ano de fundação. O Aragipe Praia Hotel e a Pousada Abaís estão localizados na capital, Aracaju, enquanto o Xingó Parque Hotel se situa na cidade de Canindé do São Francisco. De acordo com Sebrae (2012), a Pousada Abaís e o Xingó Parque Hotel são classificados como pequena empresa, e o Aragipe Praia Hotel é considerado microempresa. Todas as empresas foram fundadas na década de 1990.

Tabela 1 - Informações referentes às empresas

\begin{tabular}{llcc}
\hline \multicolumn{1}{c}{ Empresa } & Local & No de Funcionários & Ano de Fundação \\
\hline Aragipe Praia Hotel & Capital & 8 & 1991 \\
Pousada Abaís & Capital & 10 & 1997 \\
Xingó Parque Hotel & Interior & 49 & 1991 \\
\hline
\end{tabular}


No Xingó Parque Hotel, entrevistou-se a proprietária do estabelecimento. Já no Aragipe Praia Hotel, o entrevistado foi o gerente e também herdeiro, já que sua genitora é a proprietária. Na Pousada Abaís, foi possível entrevistar a proprietária (mãe) juntamente com a gerente (filha).

\subsubsection{Equipamentos e ferramentas de TI existentes}

Nesta categoria de análise foram explorados os elementos: equipamentos de TI implantados e ferramentas de TI instaladas. Tais elementos são necessários para conhecer a realidade dos participantes com relação às tecnologias utilizadas pela empresa e às disponibilizadas aos clientes. Os meios de hospedagem analisados possuem computadores, e todos são conectados à Internet. Além disso, disponibilizam Internet sem fio para seus hóspedes de forma gratuita.

O Xingó Parque Hotel possui onze computadores em atividade nos diversos setores da empresa com um sistema de gestão integrado, que abrange as áreas administrativa, de faturamento, financeira, tesouraria, entre outras. A Pousada Abaís possui um computador na recepção e outro na gerência, porém o sistema utilizado para a gestão encontra-se instalado somente no computador da recepção. $O$ Aragipe Praia Hotel faz o uso de um notebook apenas para o controle de e-mail, ou seja, a gestão ainda é realizada de forma manual.

Percebe-se um número crescente de micro e pequenas empresas que estão utilizando seus sites como ferramentas de apoio à gestão, principalmente para prestar atendimento ao cliente. Essa mudança pode provocar a rotinização cada vez maior de processos por meio da Internet (Dholakia \& Kshetri, 2004).

Os hotéis e pousada participantes desse estudo possuem site, porém verificaram-se algumas deficiências com destaque para o não uso do serviço de reserva on-line. Contudo, apesar de benefícios como maior agilidade e mais segurança para o cliente (Thomaz et al., 2013), esse serviço pode gerar problemas se o sistema de gestão não estiver programado para tal, o que foi explicado pela proprietária do Xingó Parque Hotel:

[O site] ainda não está ligado ao meu sistema, porque isso me atrapalha. Como é um hotel pequeno, de 60 apartamentos, se o cara disser: "Eu quero a reserva para tal dia". E ele fizer a reserva direto no meu sistema, isso vai me atrapalhar. Porque eu tenho várias reservas, eu tenho contrato, então eu não posso deixar que o cliente faça a reserva dentro do meu sistema. Ele manda para mim a solicitação. A solicitação entra nesse computador, que é exclusivo de reservas. A gente verifica se tem disponibilidade, responde ao cliente, o cliente confirma, e a gente coloca no sistema.

A Pousada Abaís também não adota a reserva de forma on-line, pois, segundo a proprietária, os valores referentes às diárias não são publicados no site. Já o gerente do Aragipe Praia Hotel destaca o uso do telefone para a realização da reserva, pois entende que o site é útil para que o hóspede conheça as instalações do hotel.

Além dos sites, os clientes têm feito o uso crescente de redes sociais tanto para a comunicação quanto para diminuir os riscos de aquisição de serviços inexistentes ou sem qualidade (Thomaz et al., 2013; Atadil et al., 2010). Dessa forma, as redes sociais tornaram-se um potencial instrumento para que hotéis e pousadas conquistem novos clientes e mantenham um relacionamento direto e duradouro com seus hóspedes.

Nesse sentido, foi observado que o Facebook é a rede social mais utilizada. Na Pousada Abaís, a gerente relatou a crescente demanda por parte dos clientes usuários do Facebook e a não exploração do 
Twitter: "a demanda vem crescendo. Até porque tem pouco tempo que temos o Facebook, apenas sete meses. Porém, apesar de possuirmos conta no Twitter, nunca o utilizamos por não ter alguém que saiba manipulá-lo".

Verificou-se que o Xingó Parque Hotel possui perfis no Facebook, Twitter e YouTube. Para tanto, a gestão dos perfis deste hotel nas redes sociais é realizada por terceiros, como esclarece sua proprietária: "Temos Facebook, Twitter. Sempre atualizado, sempre ativo. Temos uma pessoa que nos presta o serviço de monitorar o site e as redes sociais. Principalmente o Facebook, pois o Twitter são mais os amigos que nos acompanham". Em particular, o perfil do Xingó no YouTube possui vídeos, mas não é atualizado há quase dois anos. A proprietária explica que as pessoas, no Brasil, ainda não exploram todo o potencial do YouTube, o que implica na sua utilização apenas como uma ferramenta de entretenimento.

O entrevistado do Aragipe Praia Hotel ainda não sentiu a necessidade do uso de redes sociais como mais uma ferramenta de comunicação e marketing. Raposo e Pereira (2012) verificaram que as preferências dos consumidores não são sempre consideradas nas estratégias de marketing e vendas dos serviços hoteleiros.

As características encontradas nos meios de hospedagem participantes foram semelhantes às verificadas no estudo de Flores, Cavalcante e Raye (2012), cujo resultado mostra que as redes sociais têm sido utilizadas apenas no relacionamento com os clientes, tendo uma tímida participação na promoção de produtos e serviços. Esses autores identificaram q

\subsubsection{Dependência da ti}

Nesta categoria de análise foram explorados os elementos: (i) acesso a informações chave para o negócio, (ii) disponibilização de informações para clientes; (iii) possibilidade de realizar novos negócios e (iv) novos canais de comunicação com clientes. As diversas ferramentas disponibilizadas pela TI facilitam o constante contato entre empresa e cliente (Farias et al., 2011; Poon, 1988; Buhalis, 1998). As reservas, antes realizadas em grande parte via telefone, hoje podem ser efetuadas por diversos canais de comunicação (Farias et al., 2011). Além disso, essas ferramentas facilitam novos contratos com fornecedores e parceiros.

No Xingó Parque Hotel a entrevistada destacou: "podemos dizer que, hoje, é $50 \%$ pelo formulário de contato [e-mail] presente no site. E entre o telefone e as redes sociais, é mais por telefone. Nosso cliente entra mais em contato devido à indicações, então o telefone é muito utilizado". Situação semelhante acontece na Pousada Abaís e no Aragipe Praia Hotel, onde o e-mail se tornou uma ferramenta tão importante quanto o telefone.

A partir dos dados coletados, pode-se classificar os meios de hospedagem analisados nos três primeiros estágios de adoção de TI propostos por Ma, Buhalis e Song (2003). O Aragipe Praia Hotel está no estágio de processamento de dados, pois a TI é utilizada apenas para a disponibilização de informações sobre o hotel e para concretizar as reservas via e-mail. A Pousada Abaís se encontra no estágio de gestão em razão da utilização de um sistema, que gerencia as reservas e o uso dos apartamentos, assim como a cobrança automática do uso do telefone e a geração de fatura para empresas. Já o Xingó Parque Hotel está presente no estágio de estratégia porque, além da disponibilização de informações, comunicação e gestão do uso e das reservas, realiza periodicamente o mapeamento do serviço para a tomada de decisão, como explica a entrevistada: 
No sistema eu tenho um setor de estatística, que me informa um resumo da ocupação, consulta de hospedagem, ocupação das empresas que são cadastradas com a gente, relatório da EMBRATUR, ocupação por período, gráficos... Por exemplo, saber o estado que me mandou mais. Todo mês eu fecho relatório. Então, a gente pega os relatórios de 3 anos pra cá e a gente vê onde é que a gente tem que atuar mais.

O uso cada vez maior da tecnologia também tem exigido maior atenção dos gestores na análise e planejamento das estratégias de adoção de TI para a disponibilização de novos serviços aos clientes (Mondo et al., 2010; Thomaz et al., 2013; Dholakia \& Kshetri, 2004). Como relatado, os hotéis e pousadas participantes deste estudo disponibilizam Internet sem fio. Esse serviço é considerado por alguns hóspedes como item indispensável, pois a escolha do local pode estar associada à oferta ou não desse serviço, como já ocorrido na Pousada Abaís e relatado pela gerente: "Tivemos um caso que alguns hóspedes já saíram por problemas na Internet. Se a Internet cair, mesmo que não seja um problema nosso, a pessoa arruma a mala e sai".

Os entrevistados tiveram percepções semelhantes sobre a importância que a TI tem para a organização quando questionados sobre uma possível indisponibilidade da Internet ou dos computadores. O gerente do Aragipe Praia Hotel entende que teria impacto em razão da mudança que está ocorrendo do uso do fax para o e-mail:

No início, antes de ter esse uso constante do computador, utilizávamos fax para receber os comprovantes de depósito. Mas agora é mais via e-mail. O fax está sendo outra via, mas as pessoas utilizam mais a Internet. Com certeza [a indisponibilidade] teria um impacto porque aumentou muito o movimento por causa da implantação do site.

Na Pousada Abaís, a entrevistada comentou que, sem o uso da TI, seria difícil trabalhar:

Seria muito caótico. Iria impactar em $80 \%$ do nosso trabalho. Além do nosso sistema não funcionar sem Internet, os hóspedes também não ficam se não tiver Internet. É o primeiro questionamento de quem vem se hospedar: "Tem Internet? É sem fio? É pago?", senão nem entram.

Apesar de possuir escritório em Aracaju e Canindé do São Francisco localizar-se a uma distância de mais de 200 quilômetros, o impacto para o Xingó Parque Hotel, segundo a proprietária, não seria tão grande. $O$ hotel utiliza medidas de prevenção e de contorno, além de possuir um funcionário específico para $\mathrm{TI}$ e de o sistema de gestão funcionar independentemente da Internet, conforme relatado:

\footnotetext{
Além de cadastrar no sistema, nós realizamos a impressão de todas as nossas reservas. A gente não confia, porque a gente já teve problemas sérios. Ficamos sem sistema, caiu o sistema. Para o técnico chegar lá era demorado. Hoje já temos uma pessoa lá de $\mathrm{TI}$, dentro do hotel, específico pra TI, fica uma pessoa lá direto. Então depois que ele foi trabalhar no hotel, não tivemos mais nada. Além disso, um relatório do ano inteiro é impresso diariamente, e é realizado um backup. O sistema lá roda sem Internet, lá o sistema é em rede. Quando a Internet cai, a comunicação daqui [Aracaju] para lá [Canindé do São Francisco] é realizada por telefone.
}

Além disso, a proprietária explica que o Xingó Parque Hotel é favorecido pela quantidade de computadores disponíveis, pois um computador pode ser substituído por outro reserva caso ocorra algum problema: 
Se, por exemplo, o computador da recepção queimar, eu posso colocar qualquer outro lá, que ele vai me dar o sistema. Todos os computadores têm o sistema instalado. Então se der algum problema, o computador é substituído na hora.

A TI ainda possibilita que essas empresas concretizem diversas parcerias por terem facilitado o aumento dos canais de distribuição e dos intermediários que oferecem serviços turísticos ( $\mathrm{O}^{\prime}$ Connor \& Frew, 2002). Por exemplo, o Aragipe Praia Hotel possui uma parceria que possibilita ao cliente realização da reserva diretamente no site do parceiro e este envia automaticamente um e-mail para o gestor informando a reserva, o que aumentou a demanda de reservas. Nesse sentido, os canais de distribuição virtuais auxiliam tanto na gestão quanto na divulgação dos meios de hospedagem.

No caso do Xingó Parque hotel, geralmente essas parcerias são consolidadas por meio de contratos com empresas que vendem pacotes turísticos on-line e com a garantia de um número pré-definido de apartamentos. Na Pousada Abaís, a sistemática é semelhante, como comenta a proprietária:

Somente acima daquele número é que eles consultam para saber se temos vagas. Mas até aquele número que a gente garantiu, somos obrigados a acatar. E se a gente ficar sem Internet, fazemos o quê? Se não tivermos o número de apartamentos disponíveis, temos que informar com antecedência que estamos lotados.

A depender do uso e do sucesso ou não da adoção, a TI pode impactar positiva ou negativamente nos custos e na melhoria do serviço para o cliente (Hameed \& Counsell, 2012; Nandan, 2009) e, por isso, é importante que os gestores planejem a aquisição, a implantação e o uso dos equipamentos de $\mathrm{TI}$ (Ghobakhloo et al., 2012; Lunardi et al., 2010). Nas empresas analisadas é possível verificar alguns pontos semelhantes, porém outros divergentes. Na Pousada Abaís e no Xingó Parque Hotel foi possível reduzir os custos com o uso de ferramentas e equipamentos de TI. A Pousada Abaís teve uma redução no uso de tinta, papel e, principalmente, tempo, como é explicado pela sua proprietária:

Antigamente, havia gasto com papel para o envio dos boletos, gastávamos papel, gastávamos tinta, mandava fazer envelope, pagava para fazer envelope timbrado, ia pro correio, isto é, tínhamos que nos deslocar. Então pagávamos os correios, e às vezes extraviava... a pessoa não recebia. Você tinha que emitir nova nota fiscal, e hoje não tem mais isso.

O Xingó Parque Hotel utiliza computadores desde a inauguração em 1991, e sua proprietária compara o uso de $\mathrm{TI}$ no hotel naquela época com o momento atual para explicar os benefícios proporcionados:

Logo no início [1991] não era esse sistema atual, era um sistema precário. Era aquela coisa, estava começando a informatização na hotelaria (...) Hoje em dia é mais fácil gerenciar. Antigamente era tudo na mão. Quando o hóspede saía, você ia somar tudo (risos). Precisávamos de mais de 30 minutos para realizar o checkout. Hoje em dia é só clicar no botão, é rápido. Isso aqui é a coisa mais rápida do mundo! Impressionante como a tecnologia é fantástica. Eu tenho um relatório diário do que foi gasto, do que não foi gasto, nota fiscal, impostos... Então é a maior facilidade do mundo.

Porém, apesar dos diversos benefícios, a dependência que a empresa cria com a tecnologia pode gerar transtornos com os clientes, como já ocorreu na Pousada Abaís no momento do checkout: 


\begin{abstract}
Vamos supor que o sistema entra em "pane". A gente fala para o hóspede: "Olha, o sistema está em pane e nós não temos como emitir a nota". Às vezes o site da própria Secretaria da Fazenda está parado, ou seja, não emite a nota. A gente não consegue emitir, quer dizer, não é a gente... Pronto! Outro ponto negativo: falta de compreensão do hóspede. E a gente faz o quê? Não podemos fazer nada... A gente passa muito por isso. Com cartões de crédito também, pois também dependem [da TI]... O sistema está fora do ar, não é? Então o hóspede nos diz: "O problema é seu, não é? Eu estou querendo pagar!". É assim que respondem.
\end{abstract}

Para o gestor do Aragipe Praia Hotel, o uso da TI não teve impacto direto na melhoria do serviço para o cliente, pois afirma que: "naturalmente tendemos a melhorar o serviço independente de tecnologia".

\title{
4.1.3 Experiência dos gestores com ti
}

Procurou-se identificar os perfis por meio do conhecimento do gestor com relação à $\mathrm{Tl}$ e o seu envolvimento nos processos anteriores de adoção de TI. Os gestores de micro e pequenas empresas têm alto poder de decisão para a adoção ou não de TI e quanto maior o seu conhecimento e habilidade com tecnologia, maior a probabilidade da decisão pela adoção (Gargallo-Castel \& Ramírez-Alesón, 2007; Grandon \& Pearson, 2004; Hameed \& Counsell, 2012; Zilber \& Araújo, 2012; Ghobakhloo et al., 2012).

No Aragipe Praia Hotel, o gestor utiliza o computador apenas para enviar e receber e-mails sobre reservas e o telefone apenas para realizar e receber chamadas. Quando ocorreu a implantação da Internet sem fio, quem acompanhou o processo foi a sua filha.

Já na Pousada Abaís, a proprietária era quem utilizava o computador até o momento que sua filha começou a auxiliar a gestão da pousada, como relatado: "eu fazia tudo sozinha, porque ela não trabalhava comigo, ela não morava aqui. E há um ano e três meses que ela está por aqui. Então eu passei tudo isso pra ela". Ambas possuem smartphone, a proprietária o utiliza exclusivamente para atividades pessoais, enquanto a gerente também o utiliza para o trabalho, inclusive gerencia pelo telefone o e-mail e o Facebook da Pousada. Porém, quando ocorre a instalação ou atualização de ferramentas e equipamentos de $\mathrm{Tl}$, quem acompanha esse processo é um dos recepcionistas que, segundo a proprietária, é muito eficiente:

Ele é autodidata, ele é curioso, ele ajuda a gente bastante. Algumas vezes, quando ocorre um problema na Internet, ele mesmo quem resolve. Não precisamos nem chamar um técnico. Então, tudo relacionado a isso, eu estando ou não, ou ela estando ou não, quem acompanha isso e resolve é ele.

Já a proprietária do Xingó Parque Hotel utiliza os sistemas gerenciais no computador além da comunicação com os clientes. Ela conhece bem e faz o uso de todo o sistema de gestão e tomada de decisão do hotel, além de elaborar relatórios gerenciais em planilhas. Questionada sobre o uso de smartphones e tablets, ela comentou:

Smartphone, eu estou aprendendo. Tablet, eu tenho, mas não uso (risos). Dou para meus filhos, mas eu mexo um pouquinho. Já tenho uma experiência. Gostaria de aprender mais. Eu não sei mexer direito, mas eu aprendo. 
Quando ocorre a instalação de um novo software no Xingó Parque Hotel, a entrevistada relatou que ela faz o acompanhamento da implantação. Mas quando acontece a adoção de um novo hardware, é o funcionário responsável pela TI quem acompanha.

\subsubsection{Dificuldades na adoção de ti}

Com relação às dificuldades na adoção de $\mathrm{Tl}$, foram investigados a escassez de recursos financeiros, a falta de mão de obra qualificada e a resistência a mudanças. Zagheni e Luna (2011) e Lam, Cho e Qu (2007) destacam que os gestores podem e devem lidar com a complexidade e os problemas que surgem devido ao uso de $\mathrm{Tl}$ e que devem manter os empregados sempre em constante capacitação para lidar com as diversas ferramentas utilizadas pela organização.

Das três empresas estudadas, somente a Pousada Abaís relatou que não houve a aquisição de tecnologia por questões financeiras. A pousada sofre com os constantes problemas devido ao provedor de telefonia e Internet, mas quando surgiu um novo provedor na cidade no ano de 2012, a pousada tentou realizar a troca para tentar sanar os problemas, contudo sua central não era compatível. Além disso, o custo de uma nova central é acima de $\mathrm{R} \$ 10.000,00$ nesse mesmo porte, e então a proprietária desistiu.

Apesar de não relatarem nenhuma barreira financeira, as situações no Xingó Parque Hotel e no Aragipe Praia Hotel divergem. Verificou-se que o Xingó investiu em tecnologia desde o início de suas atividades, enquanto o Aragipe, por exemplo, faz uso de um computador de uso pessoal.

Sobre a qualificação de pessoal, a proprietária da Pousada Abaís avaliou os três funcionários que trabalham diretamente com a $\mathrm{TI}$ a partir de uma escala de zero a cinco, e atribuiu ao conhecimento deles as notas cinco, três e dois. No Xingó Parque Hotel, foi mencionado que todos os funcionários têm uma boa noção de informática, principalmente devido à pouca rotatividade. No Aragipe, apenas o gestor e a filha utilizam computadores. Quando questionados sobre resistência no uso das ferramentas, tanto o Xingó Parque Hotel quanto a Pousada Abaís não tiveram qualquer tipo de resistência por parte dos funcionários, como explica a proprietária da Pousada Abaís:

É condição pro trabalho. Hoje se você não tiver habilidade com isso [computador], nem precisa vir trabalhar, porque não irá fazer nada. Não tem como, o cara que não mexe nem no básico com informática, não tem condições de trabalhar. Ele não vai trabalhar, porque hoje não se faz nada sem computador. Absolutamente nada.

Zagheni e Luna (2011) destacam que os gestores podem e devem lidar com a complexidade e os problemas que surgem devido ao uso de $\mathrm{Tl}$ e que devem manter os empregados sempre em constante capacitação para lidar com as diversas ferramentas utilizadas pela organização.

\section{CONCLUSÃO}

O objetivo deste estudo foi analisar os fatores que afetam a adoção de TI pelas micro e pequenas empresas do setor hoteleiro do estado de Sergipe. Com relação aos equipamentos e ferramentas de TI existentes, verificou-se que as empresas participantes possuem seus computadores conectados à Internet e disponibilizam Internet sem fio de forma gratuita. 
Particularmente, quanto ao uso de softwares de gestão hoteleira, as empresas distinguem-se: enquanto o Xingó Parque Hotel possui um sistema integrado que abrange todas as áreas para a gestão do hotel, a Pousada Abaís possui o sistema instalado somente no computador da recepção para o controle de reservas e de consumo dos hóspedes. Por sua vez, o Aragipe Praia Hotel não faz uso de software para a gestão do negócio.

Apesar das empresas estudadas possuírem site, verificaram-se algumas deficiências com destaque para o não uso do serviço de reserva on-line. Observou-se também que o Facebook é a rede social mais utilizada, porém as empresas, quando a utilizam, o fazem de forma muito limitada para a promoção de seus serviços.

Quando se observa o nível de dependência, percebe-se a TI como importante ferramenta para as atividades do negócio e o impacto de sua indisponibilidade foi atribuído como alto, especialmente na Pousada Abaís cujo software de gestão está instalado em um só computador e exige uma conexão com a Internet. Além disso, houve redução de custos da Pousada Abaís e do Xingó Parque Hotel com a adoção de $\mathrm{TI}$, o que facilitou a gestão financeira. Porém, verificou-se que a dependência que a empresa cria com a tecnologia pode gerar transtornos com os clientes.

Já com relação à experiência dos gestores com TI, identificaram-se três diferentes perfis desde o uso restrito de e-mail até o domínio de todo o sistema de gestão. Quando ocorre a instalação ou atualização de ferramentas e equipamentos de $\mathrm{TI}$, geralmente quem acompanha não são os gestores, exceto no Xingó Parque Hotel quando se trata da implantação de softwares.

No que toca às dificuldades na adoção de $\mathrm{TI}$, identificou-se apenas um caso relatado de barreira financeira para a aquisição de novos equipamentos. Observou-se que o conhecimento em informática dos funcionários é indispensável e que não houve resistência em qualquer adoção.

As limitações deste estudo estão ligadas diretamente às limitações da estratégia de estudos de caso. Esse tipo de estratégia de pesquisa é limitada principalmente por não permitir a inferência de generalizações (Yin, 2001; Stake, 1994).

Para futuros trabalhos, indica-se a realização de estudos quantitativos que explorem as variáveis da adoção de TI nas MPEs, assim como estudos que identifiquem a correlação entre a adoção de TI e o aumento ou a redução de turistas nos meios de hospedagem, e o impacto da TI nos custos dessas empresas.

\section{REFERÊNCIAS}

ABIH/SE. (2012). Sergipe registra maior média de ocupação hoteleira de todos os tempos. Acesso em 26 de 11 de 2012, disponível em http://www.abih-se.com.br/noticias:1265/sergipe-registramaior-media-de-ocupacao-hoteleira-de-todos-os-tempos.html.

$$
20(3), \text { pp. 427-445. }
$$

, C. (2010). An Analysis of the Usage of

$$
\text { Facebook and Twitter as a Marketing Tool in Hotels. ， } 11 \text { (2), pp. 119-125. }
$$


Barbosa, L. G., O'neil, I., \& Marins, C. T. (2005). British Travellers' image perspectives of Brazil as a tourism destination. ENCONTRO NACIONAL DOS PROGRAMAS DE PÓS-GRADUAÇÃO EM ADMINISTRAÇÃO, 29. Brasília.

Barbosa, M. d., Souza, A. d., FARIAS, S., \& Kovacs, M. (2013). Decodificando websites: Como criar uma imagem mental distintiva para os serviços de hotelaria? Revista Brasileira de Pesquisa em Turismo , 7 (1), pp. 114-128.

Bezerra, E. D., Luft, M. C., \& Dacorso, A. L. (2012). E

Estudios y Perspectivas en Turismo, 21, pp. 1262-1280.

Buhalis, D. (1998). Strategic use of information technologies in the tourism industry. Tourism Management, 19 (5), pp. 409-421.

Buhalis, D., \& Law, R. (2008). Progress in information technology and tourism management: 20 years on and 10 years after the Internet-The state of eTourism research. Tourism Management, 29, pp. 609-623.

Cacho, A. d., \& Azevedo, F. F. (2010). O Turismo no Contexto da Sociedade Informacional. Revista Brasileira de Pesquisa em Turismo , 4 (2), pp. 31-48.

Chagas, M.M., Marques Junior, S., \& Duarte, A.C.F. (2013). : um estudo em Canoa Quebrada/CE. [ösi:Revista

Brasileira de Pesquisa em Turismo, 7 (3), pp. 456-475.

\section{- SP. ENANPAD, Encontro da Associação}

Nacional dos Programas de Pós Graduação em Administração. 29. Brasília: Anais...

Dholakia, R. R., \& Kshetri, N. (2004). Factors Impacting the Adoption of the Internet among SMEs. Small Business Economics, 23, pp. 311-322.

Easterby-Smith, M., Thorpe, R., \& Lowe, A. (1999). Pesquisa Gerencial em Administração. São Paulo: Pioneira.

Eisenhardt, K. M. (1989). Building Theories from Case Study Research. Academy of Management Review, 14, pp. 532-550.

EMSETUR. (2012). Pesquisa de Demanda Turística.

Farias, S. A., Filho, M. J., Barbosa, M. d., \& Costa, C. S. (2011). Experiência extraordinária na internet? Uma análise da oferta de experiência em portais de turismo governamentais. REGE, 18 (3), pp. 451-468. 
(SC, Brasil). Revista Brasileira de Pesquisa em Turismo , 6 (3), pp. 62-79.

Gargallo-Castel, A., \& Ramírez-Alesón, M. (2007). La Adopcion de las Tecnologias de la Informacion en las Pequenas Y Medianas Empresas. Revista Alcance, 14 (3), pp. 357-374.

Ghobakhloo, M., Hong, T. S., Sabouri, M. S., \& Zulkifli, N. (2012). Strategies for Successful Information Technology Adoption in Small and Medium-sized Enterprises. Information , 3.

Gouvêa, M. A., Niño, F. M., \& Mantovani, D. M. (2012). Intenção de adoção da Internet para a aquisição de serviços turísticos. Revista Alcance , 19 (4), pp. 476-496.

Grandon, E. E., \& Pearson, J. M. (2004). Electronic commerce adoption: an empirical study of small and medium US businesses. Information and Management , 42, pp. 197-216.

Hameed, M. A., \& Counsell, S. (2012). Assessing the Influence of Environmental and CEO Characteristics for Adoption of Information Technology in Organizations. Journal of Technology Management \& Innovation, 7 (1), pp. 64-84.

IBGE. (2010). Pesquisa Anual de Serviços (Vol. 12). Rio de Janeiro.

IBGE. (2012). Pesquisa de Serviços de Hospedagem. Rio de Janeiro.

Lam, T., Cho, V., \& Qu, H. (2007). A study of hotel employee behavioral intentions towards adoption of information technology. Hospitality Management , 26, pp. 49-65.

Laville, C., \& Dionne, J. A. (1999). Construção do Saber: Manual de Metodologia da Pesquisa em Ciências Humanas. Belo Horizonte: Editora da UFMG.

Lunardi, G. L., Dolci, P. C., \& Maçada, A. C. (2010). Adoção de tecnologia de informação e seu impacto no desempenho organizacional: um estudo realizado com micro e pequenas empresas. Revista de Administração, 45 (1), pp. 05-17.

Ma, J. X., Buhalis, D., \& Song, H. (2003). ICTs and Internet adoption in China's tourism industry. International Journal of Information Management , 23, pp. 451-467.

caso da Ilha da Madeira. Revista Turismo em Análise , 19 (1), pp. 25-42.

Mondo, T. S., Santos, F., \& Costa, J. I. (2010). Os websites vistos da perspectiva do cliente: um estudo na hotelaria. Observatório de Inovação do Turismo , 5.

Nandan, S. (2009). Adoption of Information and Communication Technology in Small and Medium Enterprises: A Synthesis of Literature. Sri Lankan Journal of Management, 14 (2), pp. 18-40. 
Neuman, W. L. (1997). Social Research Methods: qualitative and quantitative aproach. Boston: Allyn \& Bacon.

O'connor, P., \& Frew, A. J. (2002). The Future of Hotel Electronic Distribution: Expert and Industry Perspectives. Cornell Hotel and Restaurant Administration Quarterlv .

turismo. , 5 (4).

Poon, A. (1988). Tourism and Information Technologies. Annals of Tourism Research, 15, pp. 531549.

Raposo, W. G., \& Pereira, R. C. (2012). CALIDAD DE LOS SITIOS WEB EN LA PERCEPCIÓN DE LOS USUARIOS: Una aplicación en los hoteles de João Pessoa, Paraíba, Brasil. Estudios y Perspectivas en Turismo, 21, pp. 925-944.

Saunders, M., Lewis, P., \& Thornill, A. (2007). Research Methods for Business Students (4 ed.). Harlow, England: Pearson Education.

SEBRAE. (2012). Anuário das Pesquisas sobre as Micro e Pequenas Empresas (2011). Série Estudos e Pesquisas.

Souza, A. G., Barbosa, M. d., Kovacs, M. H., \& Marques, R. Q. (2012). PERCEPCIÓN DEL RIESGO EN LA ELECCIÓN DE SERVICIOS HOTELEROS EN EL ÁMBITO VIRTUAL: Actitud de los consumidores frente al uso de estrategias de reducción de riesgos. Estudios y Perspectivas en Turismo , 21, pp. 52-67.

pp. 75-89.

. Revista Brasileira de Pesquisa em Turismo , 6 (2),

Stake, R. E. (1994). Case Studies. In: N. K. Denzin, \& Y. S. Lincon, Handbook of Qualitative Research (pp. 236-247). London: SAGE Publications.

Tan, B. S. (2004). The Consequences of Innovation. The Innovation Journal: The Public Sector Innovation Journal , 9 (3), pp. 764-764.

Thomaz, G. M., Biz, A. A., \& Gândara, J. M. (2013). Innovación en la Promoción Turística en Medios y Redes Sociales: Un estudio comparativo entre destinos turísticos. Estudios y Perspectivas en Turismo, 22, pp. 102-119.

Vergara, S. C. (2008). Métodos de Pesquisa em Administração. São Paulo: Atlas. 
Yang, X., \& Fu, J. (2008). Review of IT/IS Adoption and Decision-Making Behavior in Small Businesses. Tsinghua Science \& Technology, 13 (3), pp. 323-328.

Yin, R. K. (2001). Estudo de Caso: Planejamento e Métodos. Porto Alegre: Bookman.

: Um Panorama da Realidade Nacional.

, $11(2)$, pp. 476-502.

Zilber, S. N., \& Araújo, J. B. (2012). Small Companies Innovations in Emerging Countries: E-Business Adoption and its Business Model. Journal of Technology Management \& Innovation, 7 (2), pp. 102-116.

Artigo recebido em: 28/05/2013. Artigo aprovado em: 18/03/2014. 